\title{
The application of optical coherence tomography in neurologic diseases
}

\author{
Ramiro S. Maldonado, MD* \\ Pradeep Mettu, MD* \\ Mays El-Dairi, MD \\ M. Tariq Bhatti, MD
}

\begin{abstract}
Optical coherence tomography (OCT) has become an increasingly popular tool in various disciplines of medicine, particularly ophthalmology and neurology. It is an imaging technology that has revolutionized the practice of ophthalmology by providing anatomic detail of pathologic changes in the retina and optic nerve. OCT is routinely used as an ancillary test that can aid in the diagnosis and monitoring of neuroophthalmic diseases such as papilledema, optic neuritis, and neuroretinitis. OCT measurements have also been shown to predict visual prognosis in compressive optic neuropathies. Changes in OCT measurements have been used to study the course of particular

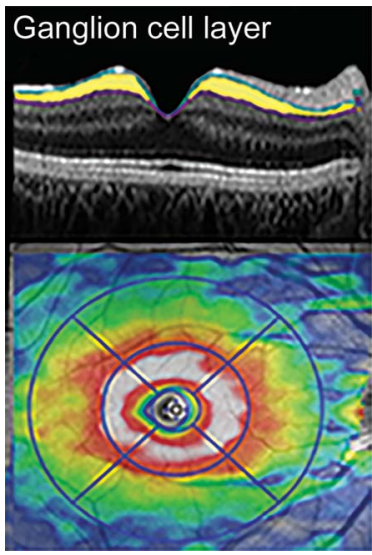
neurologic diseases such as multiple sclerosis, suggesting that the data obtained may be useful as a biomarker in diagnosing and treating neurodegenerative disease. We present an up-to-date review of the OCT findings in several diseases of neurologic interest and provide clinical examples pertinent to the general neurologist. Neurol Clin Pract 2015;5:460-469
\end{abstract}

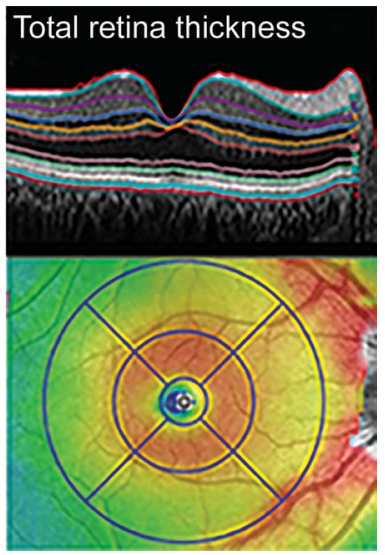

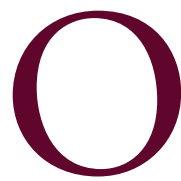

ptical coherence tomography (OCT) is a noncontact, nonradiation, fast, efficient, safe, and reproducible diagnostic imaging tool that provides histologic information on neural tissue in vivo. ${ }^{1}$ OCT was first described in 1993 by Swanson et al. $^{2}$ as a system capable of measuring different reflective properties of the retinal layers in the antemortem human eye. The first commercial OCT system was introduced in 1996, allowing for a better understanding of glaucomatous and retinal diseases. In 2001, a second-generation OCT system was developed, providing higher resolution images with faster acquisition time. ${ }^{3}$ This system is known as spectral-domain OCT, and it is the one currently available in the office setting under different commercial names. It provides

*These authors contributed equally to this work.

Department of Ophthalmology (RSM, PM, ME-D, MTB), Duke University Eye Center; and Department of Neurology (MTB), Duke University School of Medicine, Durham, NC.

Funding information and disclosures are provided at the end of the article. Full disclosure form information provided by the authors is available with the full text of this article at Neurology.org/cp.

Correspondence to: tariq.bhatti@duke.edu 


\section{CNS demyelination in neuromyelitis optica typically follows a more aggressive and debilitating course compared to MS. Distinguishing between these 2 diseases has important prognostic and therapeutic implications.}

cross-sectional images of the macula and optic nerve head with enough resolution to differentiate all retinal layers and subcellular photoreceptor elements. The different commercial systems differ essentially in the data output generated by their proprietary software. Most systems provide instant measurements of distinct layers with progression analysis within visits of a same patient and comparison of the patient's measurements to a normative database (table e-1 and figure e-1 at Neurology.org/cp).

\section{Images of interest for neurology: The normal macula and optic nerve}

The ocular structures of interest for assessment in neurologic diseases are the macula and the optic nerve. The macular images obtained by OCT demonstrate the architecture of the various retinal layers at the cellular level. ${ }^{4}$ These layers have specific reflectivity profiles (figure 1). With the nuclear layers being hyporeflective (black) and the axonal layers being hyperreflective (white), OCT analysis is preferentially done by studying volumetric changes referenced as color thickness maps. The color thickness maps can be computed at the macula (figure 1, top right) and also around the optic nerve (figure 1, bottom right).

\section{OCT findings in neuro-ophthalmic and neurologic diseases}

OCT serves as a way to quantify structural changes of the optic nerve and retina that can be followed over time, which can be informative about the natural history of the disease. OCT findings in specific neuro-ophthalmic and neurologic diseases are summarized in the following sections.

Multiple sclerosis Multiple sclerosis (MS) causes both demyelination and axonal loss through a complex and incompletely understood inflammatory and neurodegenerative process. The peripapillary retinal nerve fiber layer (RNFL) thickness can be used to assess axonal loss. Prior to exiting the lamina cribrosa, the retinal ganglion cell axons (which comprise the RNFL) are unmyelinated, thereby allowing OCT measurements to avoid any confounding due to myelin and pathologic processes affecting myelin. More recently, as investigators have been able to segment the various layers of the retina, the ganglion cell layer (GCL) thickness from macular scans has been studied to directly quantify neuronal loss, which may be more accurate than measurements of total macular volume. ${ }^{5}$ Microcystic retinal edema without a clinically identifiable cause can be seen in the inner nuclear layer (INL) in patients with MS, and this may independently affect visual function. ${ }^{6}$

In patients with MS who have not experienced a clinical episode of optic neuritis, the average peripapillary RNFL thickness has been shown to be thinner than in healthy controls. ${ }^{7}$ Even in the absence of a symptomatic episode of optic neuritis, RNFL thickness correlates well with visual function measures, including low-contrast visual acuity and contrast sensitivity. ${ }^{8}$ Similarly, in patients with MS who have not experienced an episode of optic neuritis, whole brain, gray matter, and white matter volumes have been shown to correlate with both RNFL thickness and GCL thickness. ${ }^{9}$ OCT can be used to determine if the rate of neuronal or axonal loss can be delayed with the institution of disease-modifying drugs. ${ }^{10}$
Supplemental Data

Neurology.org/cp 
Figure 1 Retinal layer segmentation
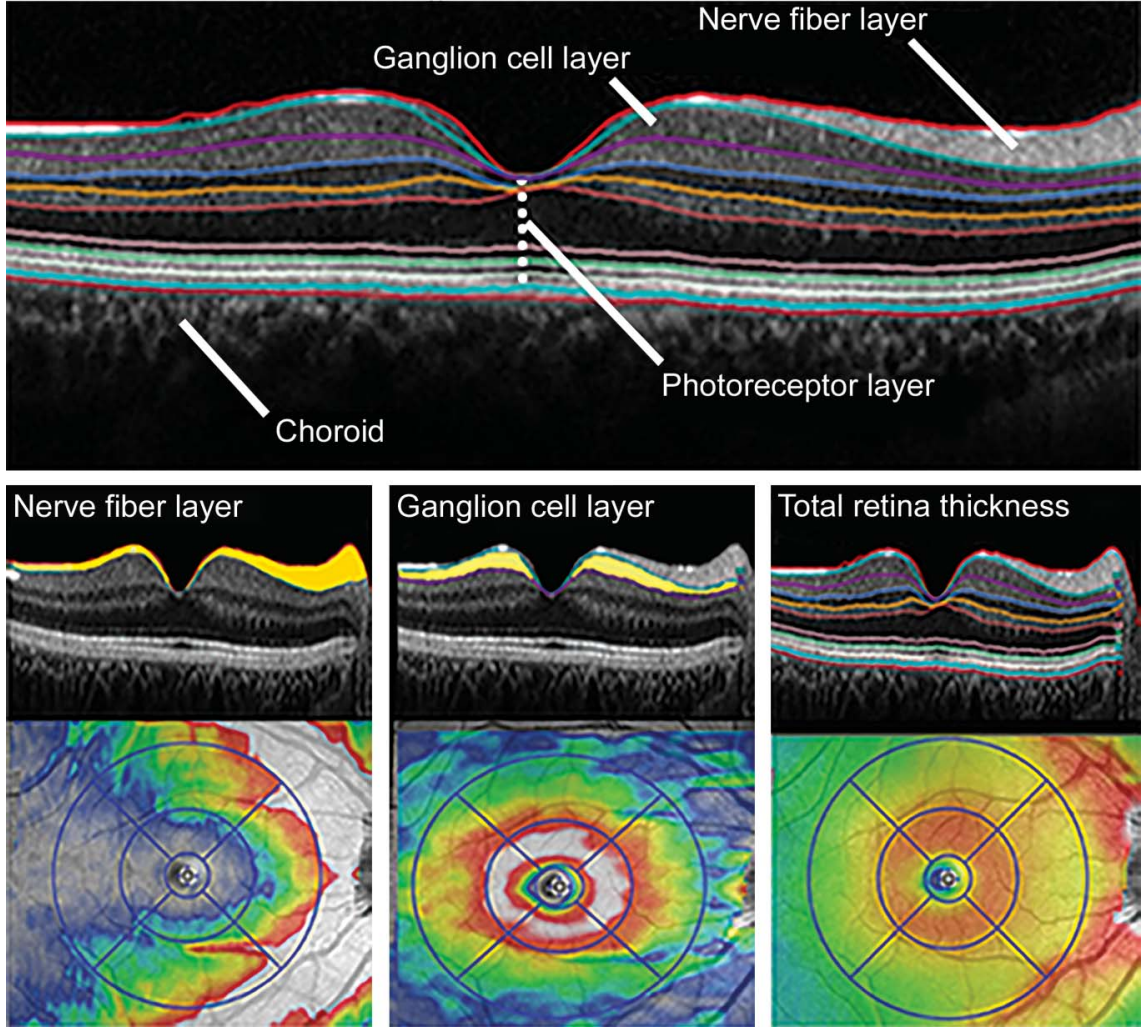

Images shown were obtained with Spectralis spectral-domain optical coherence tomography (SD-OCT) system (Heidelberg Engineering, Germany). Top panel is a cross-sectional SD-OCT scan at the fovea that shows all the retinal layers segmented. The most relevant to study neurologic diseases are labeled. From top to bottom, the segmented retinal layers are as follows: internal limiting membrane, nerve fiber layer, ganglion cell layer, inner plexiform layer, inner nuclear layer, outer plexiform layer, external limiting membrane, myoid zone, ellipsoid zone, retinal pigment epithelium, Bruch membrane complex. Bottom panels depict the segmented layer with the corresponding color thickness map plotted below.

Analysis of the segmented retinal layers has shown that while there is generalized atrophy of the inner retinal layers in patients with MS, the GCL and inner plexiform layer (IPL) are preferentially affected. ${ }^{11}$ Combined GCL and IPL thickness has been shown to be superior to RNFL thickness in correlations with scores from the Expanded Disability Status Scale, visionrelated quality of life metrics, as well as high- and low-contrast visual acuity testing. ${ }^{12}$

The progressive thinning of retinal layers as measured by OCT varies depending on the subtype of MS. In eyes with optic neuritis, when compared to clinically isolated syndrome, both secondary progressive MS and relapsing-remitting MS had significantly thinner measurements. ${ }^{13}$

Acute demyelinating optic neuritis OCT has been used to study the presentation and progression of acute idiopathic inflammatory demyelinating optic neuritis (figure 2). The peripapillary RNFL is thickened even in cases in which the optic nerve appears clinically normal. ${ }^{14}$ Changes can be reliably detected as early as 2 months following an acute episode of optic neuritis and the RNFL thickness typically stabilizes by 6 months though minor changes can happen thereafter. ${ }^{15}$ Measurements of the RNFL prior to 2 months may not be an accurate representation of axonal loss due to unresolved RNFL edema. ${ }^{15}$ Investigators have shown that the GCL does not swell acutely and can be followed more accurately over time for the presence of thinning indicative of neuronal loss. ${ }^{16}$ Thinning of the GCL can be detected by 1 month, and combined GCL + IPL thickness decreases before RNFL thinning in eyes with optic neuritis. ${ }^{17}$ Eyes with a previous remote episode of optic neuritis have diffuse 


\section{OCT is particularly useful clinically in papilledema as it can be used to see how optic nerve structure changes over time in response to treatment.}

thinning of the peripapillary RNFL with preferential involvement of the temporal quadrant. ${ }^{15,18}$ While an episode of optic neuritis causes a substantial reduction in the RNFL, it is not believed to hasten the rate of progressive RNFL thinning over time due to the underlying disease process in patients with MS.?

RNFL thickness measurements in patients with optic neuritis correlates with visual function parameters including mean deviation on automated perimetry. GCL thickness is a stronger predictor of visual disability than RNFL. Patients with RNFL thickness below $75 \mu \mathrm{m}$ are more likely to have persistent visual field defects. ${ }^{19}$ RNFL thickness following an episode of optic neuritis has not been shown to be useful in predicting the future risk of developing MS. ${ }^{20}$ These OCT findings in acute demyelinating optic neuritis are clinically applicable and can aid a practitioner in diagnosis and providing prognostic information.

Neuromyelitis optica CNS demyelination in neuromyelitis optica (NMO) typically follows a more aggressive and debilitating course compared to MS. Distinguishing between these 2 diseases has important prognostic and therapeutic implications. After at least 3 months, the peripapillary RNFL is typically thinner following NMO optic neuritis compared to MS optic neuritis. ${ }^{21}$ The pattern of RNFL loss may help distinguish NMO optic neuritis from MS optic neuritis. In NMO optic neuritis, RNFL loss is more severe, diffuse, and involves the superior and inferior quadrants. In MS optic neuritis, RNFL loss is less severe and predominantly affects the temporal quadrant that contains the papillomacular bundle. Macular volumes are significantly reduced in eyes that have had an episode of NMO optic neuritis. ${ }^{22}$ Segmentation of retinal layers has also been used to demonstrate inner retinal changes following NMO optic neuritis. Eyes with a history of NMO optic neuritis have more thinning of the combined GCL-IPL than eyes of patients with NMO without a history of optic neuritis. ${ }^{16}$ Peripapillary RNFL measurements in eyes with a history of NMO optic neuritis have been shown to correlate with visual field defects, visual acuity, contrast sensitivity, visual evoked potentials, and the Expanded Disability Status Scale score. ${ }^{23}$ These findings may be clinically useful as the imaging features may lead a physician to more strongly consider a diagnosis of NMO in the appropriate patient.

Papilledema and idiopathic intracranial hypertension OCT has become a valuable tool in the care of patients with papilledema and idiopathic intracranial hypertension. For example, OCT volumetric measurements correlate well with clinical grading of optic nerve head photographs, where for Frisen scale 0 to scale 4 the total retinal volumes were 11.36, 12.53, 14.42, 17.48 , and 21.81, respectively (figure 3 ). ${ }^{24}$ OCT has also been shown to correlate with increased intracranial pressure ${ }^{25}$ and with visual field changes demonstrating a linear correlation of a decrease of $0.6 \mathrm{db}$ per each $10 \mu \mathrm{m}$ of axonal thickness increase. ${ }^{26}$ OCT is particularly useful clinically in papilledema as it can be used to see how optic nerve structure changes over time in response to treatment.

OCT can also aid in differentiation of optic nerve head drusen (i.e., pseudopapilledema) from papilledema. True papilledema has a higher head elevation, hyporeflective space at the optic nerve head, and smaller RNFL-optic nerve head angle compared to cases of pseudopapilledema due to optic nerve head drusen. ${ }^{27}$ Hyperreflective foci causing irregular elevation of the optic nerve head is suggestive of optic nerve head drusen (figure e-2). 
Figure 2 Early and late optical coherence tomography features in optic neuritis

Segmented RNFL layer
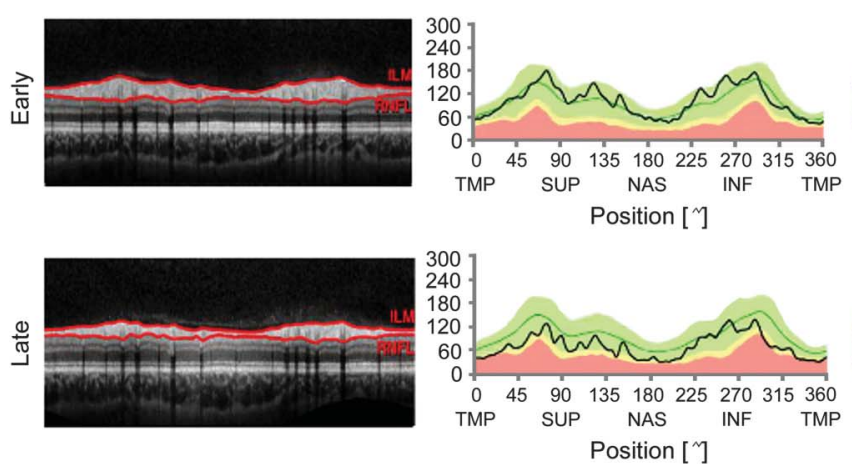

C

RNFL thickness quadrants
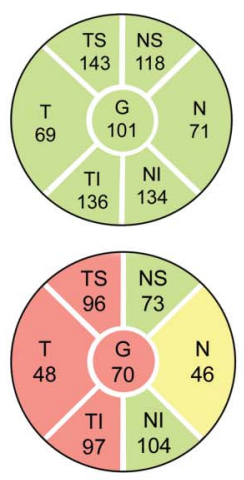

D Total retinal thickness
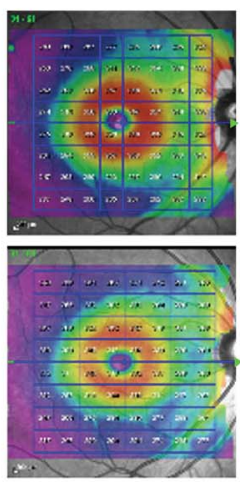

Top row: on the day of symptoms presentation with vision of 20/40. Bottom row: 4 months later with vision of hand motion. (A) System automatic segmentation, thinner in the late stage. (B) Retinal nerve fiber layer (RNFL) thickness profile compared against normative data. (C) RNFL thickness per quadrants in a circle around the optic nerve (peripapillary thickness). (D) Total retinal thickness maps that includes RNFL and ganglion cell layer. INF $=$ inferior; $\mathrm{N}=$ nasal; $\mathrm{NAS}=$ nasal; $\mathrm{NI}=$ nasal-inferior; $\mathrm{NS}=$ nasal-superior; $\mathrm{SUP}=$ superior; $\mathrm{T}=$ temporal; $\mathrm{TMP}=$ temporal; $\mathrm{TI}=$ temporal-inferior; TS $=$ temporal-superior.

Neuroretinitis Neuroretinitis is characterized by optic nerve swelling associated with macular exudates in a star configuration. Peripapillary RNFL shows thickening, as one would expect from clinical examination; however, changes in the macula distinguish neuroretinitis from other causes of unilateral optic nerve edema. Typically with neuroretinitis, the optic nerve edema is associated with a serous macular detachment, and the macular star develops between 9 and 12 days after the subretinal fluid resorbs. ${ }^{28}$ Macular OCT performed early in the disease course may demonstrate subretinal fluid, which should prompt consideration and evaluation for underlying causes of neuroretinitis (i.e., Bartonella henselae) ${ }^{29}$ Macular OCT may also be helpful in detecting subclinical subretinal fluid in the cases in which the macular star does not develop (figure e-3). ${ }^{30}$ There is often abnormal foveal contour and retinal thickening along with the subretinal fluid. ${ }^{31}$ Fluid or exudates in the outer plexiform layer may also be seen. ${ }^{32}$ Macular OCT can be critical in diagnosing a case of neuroretinitis when the macular changes are subtle on clinical examination.

Optic pathway glioma In search of a noninvasive technique to detect optic pathway gliomas (OPGs), the first study utilizing OCT was published in $2010 .{ }^{33}$ Patients with neurofibromatosis-1 (NF1) with OPGs were found to have thinner peripapillary RNFL and maculae than healthy controls, whereas patients with NF1 without OPGs had measurements that were similar to healthy controls. Eyes of most patients with OPGs (both with and without NF1) that demonstrate a reduction in peripapillary RNFL thickness also have visual field loss or decreased vision, highlighting the relationship between structure and function. ${ }^{34}$ Similarly, combined macular GCL-IPL thickness was thinner in children with OPGs and vision loss. ${ }^{\mathrm{e} 1}$ When compared to visual function assessment and optic disc evaluation, OCT measurements of the peripapillary RNFL had the highest correlation with the presence of OPGs. ${ }^{\mathrm{e} 2}$ Changes in the peripapillary RNFL over time correlate with clinical and radiologic changes in children with OPGs, making OCT clinically useful for patients with OPGs. ${ }^{\text {e3 }}$

Compressive optic neuropathy The clinical presentation of a compressive optic neuropathy depends on the location and chronicity of the lesion. Cupping of the optic nerve head can be seen in some cases of a compressive optic neuropathy. Numerous studies have sought to identify clinical features to help distinguish compressive optic neuropathy-related optic nerve head cupping from the more ubiquitous glaucomatous optic nerve head cupping. ${ }^{\text {e4 }}$ OCT can 
Figure 3 Papilledema severity as seen by confocal scanning laser ophthalmoscopy (SLO)
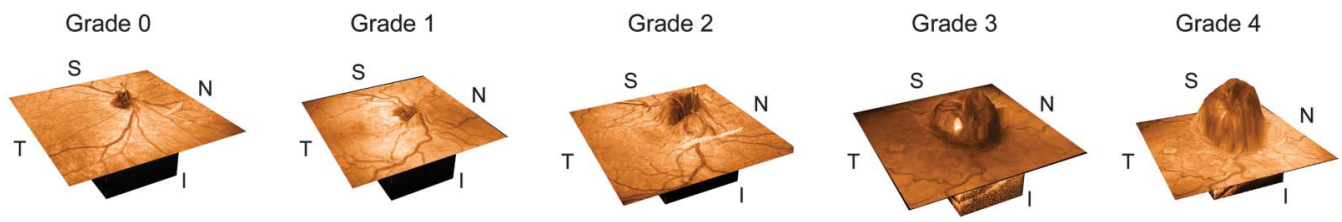

This feature, available on the Spectralis optical coherence tomography (OCT) system, utilizes a laser light to scan the retina point by point. The light reflected from the retina goes through a small aperture, which eliminates light coming from other panes, resulting in a sharp, high-resolution image. We present SLO scans corresponding to the clinical stages of the Frisen scale. Note that the scan of grade 5 could not be reliably created due to the amount of nerve swelling that goes beyond the imaging window on OCT.

differentiate compressive optic neuropathy from glaucomatous optic neuropathy. Both entities show enlargement of the optic cup and thinning of the RNFL; however, there is more thinning nasally and temporally in compressive optic neuropathy. ${ }^{\mathrm{e}}$

OCT can be used to predict the likelihood of postoperative visual recovery in compressive optic neuropathy (figure e-4). Patients with thinning (less than $97.5 \%$ of normal) of the RNFL were less likely to show any improvement in visual acuity or visual fields following resection of parachiasmal tumors. However, subjects with RNFL measurements above this threshold had significant improvement in visual acuity and improved visual fields postoperatively. ${ }^{\mathrm{e}}$ These results were confirmed in another study that demonstrated that thicker preoperative RNFL correlated with an increased likelihood of visual field recovery regardless of a patient's age or duration of symptoms. ${ }^{\text {e }}$ Similar results have been found in patients with anterior visual pathway meningiomas. ${ }^{\mathrm{e} 8}$ Patients with evidence of a chiasmopathy or an optic neuropathy who underwent surgery or fractionated stereotactic radiotherapy were more likely to have improvement in visual function if preoperative peripapillary RNFL measurements were normal. These findings have important prognostic implications in patients with compressive optic neuropathy.

Nonarteritic anterior ischemic optic neuropathy As in other cases of optic nerve swelling, OCT can help diagnose edema in nonarteritic anterior ischemic optic neuropathy (NAION), the most common cause of an acquired optic neuropathy in adults over 50 years of age. ${ }^{e 9}$ As the edema resolves, OCT can be used to quantify RNFL thinning that stabilizes at 6 months and correlates with visual function. ${ }^{9}$ B Both the degree of RNFL thinning and the anatomical location of thinning correlates with visual field defects. ${ }^{\text {e10 }}$ Macular thickness similarly correlates well with visual field sensitivity. ${ }^{\mathrm{e} 11}$ Ganglion cell complex (defined as measurement between ILM to outer boundary of the IPL) measurements have also been shown to correlate well with the severity and location of visual field loss. ${ }^{\text {e12 }}$ After at least 6 months from a NAION, visual acuity correlates with temporal RNFL thickness and central macular IPL. ${ }^{\text {el3 }}$ One month following an NAION, combined GCL + IPL measurements are significantly reduced, and the thickness correlates with visual acuity and visual field mean deviation. ${ }^{17}$ OCT can be applied in NAION for both diagnostic and prognostic purposes. These applications also allow OCT to be useful investigating novel therapies.

\section{Toxic/metabolic/drug-induced optic neuropathy}

Ethambutol In a small case series of 3 patients with ethambutol optic neuropathy, thinning of the peripapillary RNFL was predictive of poor visual recovery. ${ }^{\mathrm{e} 14}$ The temporal quadrant of the peripapillary RNFL, corresponding to the papillomacular bundle, showed the greatest average decrease in thickness in a separate study. ${ }^{\text {e15 }}$ However, it should be noted that OCT changes may not be evident at the time of presentation. ${ }^{\mathrm{e} 16}$

Vitamin $B_{12}$ deficiency OCT has shown thinning of the temporal peripapillary RNFL correlating to e serum $B_{12}$ levels. ${ }^{\text {e17 }}$ Patients with vitamin $B_{12}$ supplementation have demonstrated normalization of visual evoked potentials following supplementation, but the effect of 
supplementation on the peripapillary RNFL thickness as measured by OCT has not been reported. ${ }^{\text {e18 }}$

\section{Drug-induced retinopathy}

Vigabatrin Vigabatrin (Sabril ${ }^{\circledR}$; Lundbeck Inc., Deerfield, IL) is indicated for infantile spasms as well as other seizure disorders including simple and complex partial epilepsy. Clinically, patients experience characteristic nasal RNFL thinning prior to developing optic nerve pallor. ${ }^{\mathrm{e} 19}$ OCT has been investigated as a tool to identify structural retinal changes due to vigabatrin. In a prospective cross-sectional observational study, thinning of the peripapillary RNFL correlated with mean deviation loss on automated perimetry. The study also found that thinning of the peripapillary RNFL might also occur before changes on automated perimetry. This suggests that there is utility of OCT in screening patients for vigabatrinrelated retinal toxicity prior to the loss of visual function. . $20^{20}$

Fingolimod Fingolimod (FTY720, Gilenya ${ }^{\circledR}$; Novartis Pharma AG, Basel, Switzerland) is a sphingosine-1-phosphate (S1P) receptor modulator and the first approved oral therapy for MS. The S1P receptor is involved in the regulation of vascular permeability, which may be a part of the pathophysiology of fingolimod-associated macular edema (FAME). ${ }^{\mathrm{e} 21}$ Common OCT findings in FAME include increased central foveal thickness and hyporeflective areas affecting both the INL and ONL often within $1,000 \mu \mathrm{m}$ of the foveal center (figure e-5). ${ }^{\text {e22 }}$ FAME typically occurs within the first 3-4 months after initiation of treatment.

OCT is useful in the detection of FAME, particularly as patients may be initially asymptomatic and findings on clinical examination may be subtle. In some cases, OCT may be helpful in differentiating FAME from an attack of optic neuritis, though the latter is more commonly associated with pain with eye movements and a relative afferent pupillary defect if the fellow eye has not been previously affected. OCT also demonstrates resolution of morphologic changes of the macula with successful management of FAME.

\section{Neurodegenerative diseases}

Alzheimer disease Alzheimer disease (AD) can lead to decreased RNFL thickness, increased optic disc cupping, vascular tortuosity, and vision impairment. ${ }^{23}$ Decreased macular thickness and RNFL thinning are associated with abnormalities on electroretinogram even in patients with $\mathrm{AD}$ with normal visual acuity, normal visual fields, and normal color vision. ${ }^{224}$ More recently it has been shown that there is decreased choroidal thickness in patients with $\mathrm{AD}$ compared to healthy controls. . $^{25}$ OCT may play a role in early detection of AD but more studies are required to validate its utility as a biomarker of disease.

Parkinson disease Ophthalmologic manifestations of Parkinson disease (PD) include decreased visual acuity, decreased contrast sensitivity, prolonged visual evoked potential latency, hallucinations, altered color perception, and electroretinographic changes. ${ }^{\mathrm{e} 26}$

A systematic review of 13 case-control studies totaling 644 eyes of patients with PD and 604 eyes of healthy controls was recently performed. ${ }^{27}$ The meta-analysis found a higher prevalence of RNFL thinning at the temporal quadrant compared to other quadrants. Furthermore, another study found inner retinal layers $(\mathrm{IRL}=\mathrm{RNFL}+\mathrm{GCL}+\mathrm{IPL})$ thinning in patients with PD and decreased contrast sensitivity compared to healthy controls. ${ }^{\text {e28 }} \mathrm{A}$ correlation between IRL thinning and decreased contrast sensitivity in healthy controls was also found. Investigators enrolled 129 patients with PD and 129 healthy controls in an observational prospective study and found that GCL thickness was inversely correlated with disease duration and severity. ${ }^{\text {e29 }}$ OCT may have a role in identifying patients with PD early in the disease or in those patients who present with a paucity of symptoms or atypical clinical manifestations.

Amyotrophic lateral sclerosis Researchers found no difference in measures of RNFL, GCL, INL, and ONL between 76 patients with amyotrophic lateral sclerosis (ALS) and healthy controls. ${ }^{\text {e30 }}$ In a study of 24 patients with ALS, there were significantly lower measures of average RNFL, INL, and macular thickness compared to controls. ${ }^{e 31}$ While these findings are highly 
Table 1 Optical coherence tomography features in select neurologic diseases

\begin{tabular}{|c|c|c|c|c|}
\hline $\begin{array}{l}\text { Neurologic } \\
\text { disorders }\end{array}$ & $\begin{array}{l}\text { Disease-predictive } \\
\text { features }\end{array}$ & $\begin{array}{l}\text { Acute/active disease } \\
\text { features }\end{array}$ & Chronic disease features & $\begin{array}{l}\text { Current ongoing } \\
\text { research }\end{array}$ \\
\hline \multicolumn{5}{|l|}{ Inflammatory } \\
\hline PD, AD, ALS & $\begin{array}{l}\text { AD: RNFL and } \\
\text { choroidal thinning }\end{array}$ & RNFL thinning, IRL thinning & $\begin{array}{l}\text { GCL thickness inversely } \\
\text { correlated to disease } \\
\text { duration and severity }\end{array}$ & $\begin{array}{l}\text { ALS: INL thinning } \\
\text { predicting disease } \\
\text { outcome; PD: } \\
\text { photoreceptor layer } \\
\text { thinning }\end{array}$ \\
\hline Neuroretinitis & $\begin{array}{l}\text { Amount of intraretinal } \\
\text { hyperreflective spots } \\
\text { and subretinal fluid } \\
\text { correlated with final } \\
\text { visual acuity }\end{array}$ & $\begin{array}{l}\text { RNFL thickening, subretinal } \\
\text { hyporeflectivity }\end{array}$ & $\begin{array}{l}\text { Subretinal hyporeflectivity } \\
\text { resolves, RNFL thinning may } \\
\text { be seen in some cases }\end{array}$ & \\
\hline $\begin{array}{l}\text { Fingolimod- } \\
\text { associated } \\
\text { macular edema }\end{array}$ & No & $\begin{array}{l}\text { Hyporeflective areas in the } \\
\text { INL and ONL }\end{array}$ & Unknown & \\
\hline
\end{tabular}

Abbreviations: $A D=$ Alzheimer disease; $A L S=$ amyotrophic lateral sclerosis; $G C L=$ ganglion cell layer; $I N L=$ inner nuclear layer; $\mathrm{IPL}=$ inner plexiform layer; $\mathrm{IRL}=$ inner retinal layers; $\mathrm{MS}=$ multiple sclerosis; $\mathrm{NMO}=$ neuromyelitis optica; $\mathrm{ONL}=$ outer nuclear layer; $\mathrm{PD}=$ Parkinson disease; $\mathrm{RNFL}=$ retinal nerve fiber layer.

suggestive of nonmotor involvement and visual pathway involvement in ALS, further studies are needed to clarify the structural changes and determine the clinical relevance.

\section{Miscellaneous}

Migraine RNFL measurements have been shown to be thinner in migraineurs compared to controls. ${ }^{\text {e32 }}$ Measurements of choroidal thickness were found to be increased during an acute attack in migraine patients when compared to baseline level in the same patient and when compared to a control group. ${ }^{\text {333 }}$ Further studies are needed to determine the significance and clinical utility of these findings.

\section{CONCLUSION}

OCT offers the clinician a fast, reliable, reproducible, noninvasive method to evaluate and monitor several neurologic diseases (table 1). The ability to distinguish specific OCT findings for each disease entity can aid in the diagnosis and treatment of patients. The clinician should understand the basic principles of OCT and understand how to apply OCT in clinical practice. The applications of OCT in neurologic disease in both the clinical and research settings will only continue to expand as neurologists become more familiar with this revolutionary technology.

\section{REFERENCES}

1. Toth CA, Narayan DG, Boppart SA, et al. A comparison of retinal morphology viewed by optical coherence tomography and by light microscopy. Arch Ophthalmol 1997;115:1425-1428. 
2. Swanson EA, Izatt JA, Hee MR, et al. In vivo retinal imaging by optical coherence tomography. Opt Lett 1993;18:1864-1866.

3. Drexler W, Morgner U, Ghanta RK, Kärtner FX, Schuman JS, Fujimoto JG. Ultrahigh-resolution ophthalmic optical coherence tomography. Nat Med 2001;7:502-507.

4. Staurenghi G, Sadda S, Chakravarthy U, Spaide RF. Proposed lexicon for anatomic landmarks in normal posterior segment spectral-domain optical coherence tomography: the IN•OCT Consensus. Ophthalmology 2014;121:1572-1578.

5. Kardon RH. Role of the macular optical coherence tomography scan in neuro-ophthalmology. J Neuroophthalmol 2011;31:353-361.

6. Burggraaff MC, Trieu J, de Vries-Knoppert WA, Balk L, Petzold A. The clinical spectrum of microcystic macular edema. Invest Ophthalmol Vis Sci 2014;55:952-961.

7. Garcia-Martin E, Pueyo V, Ara JR, et al. Effect of optic neuritis on progressive axonal damage in multiple sclerosis patients. Mult Scler 2011;17:830-837.

8. Fisher JB, Jacobs Da, Markowitz CE, et al. Relation of visual function to retinal nerve fiber layer thickness in multiple sclerosis. Ophthalmology 2006;113:324-332.

9. Zimmermann H, Freing A, Kaufhold F, et al. Optic neuritis interferes with optical coherence tomography and magnetic resonance imaging correlations. Mult Scler 2013;19:443-450.

10. Talman LS, Bisker ER, Sackel DJ, et al. Longitudinal study of vision and retinal nerve fiber layer thickness in multiple sclerosis. Ann Neurol 2010;67:749-760.

11. Garcia-Martin E, Polo V, Larrosa JM, et al. Retinal layer segmentation in patients with multiple sclerosis using spectral domain optical coherence tomography. Ophthalmology 2014;121:573-579.

12. Saidha S, Syc SB, Durbin MK, et al. Visual dysfunction in multiple sclerosis correlates better with optical coherence tomography derived estimates of macular ganglion cell layer thickness than peripapillary retinal nerve fiber layer thickness. Mult Scler 2011;17:1449-1463.

13. Costello F, Hodge W, Pan YI, Freedman M, DeMeulemeester C. Differences in retinal nerve fiber layer atrophy between multiple sclerosis subtypes. J Neurol Sci 2009;281:74-79.

14. Pro MJ, Pons ME, Liebmann JM, et al. Imaging of the optic disc and retinal nerve fiber layer in acute optic neuritis. J Neurol Sci 2006;250:114-119.

15. Costello F, Hodge W, Pan YI, Eggenberger E, Coupland S, Kardon RH. Tracking retinal nerve fiber layer loss after optic neuritis: a prospective study using optical coherence tomography. Mult Scler 2008;14:893-905.

16. Syc SB, Saidha S, Newsome SD, et al. Optical coherence tomography segmentation reveals ganglion cell layer pathology after optic neuritis. Brain 2012;135:521-533.

17. Kupersmith MJ. Optical imaging of the optic nerve. J Neuroophthalmol 2015:35:210-219.

18. Wang XL, Yu T, Xia DZ, Zhang JS, Yan Q, Luo YH. Measurement of retinal nerve fiber layer thickness in optic atrophy eyes of patients with optic neuritis using optical coherence tomography. Graefes Arch Clin Exp Ophthalmol 2010;248:1013-1018.

19. Costello F, Coupland S, Hodge W, et al. Quantifying axonal loss after optic neuritis with optical coherence tomography. Ann Neurol 2006;59:963-969.

20. Costello F, Hodge W, Pan YI, Metz L, Kardon RH. Retinal nerve fiber layer and future risk of multiple sclerosis. Can J Neurol Sci 2008;35:482-487.

21. Green A, Cree B. Distinctive retinal nerve fibre layer and vascular changes in neuromyelitis optica following optic neuritis. J Neurol Neurosurg Psychiatry 2009;80:1002-1005.

22. Naismith RT, Tutlam NT, Xu J, et al. Optical coherence tomography differs in neuromyelitis optica compared with multiple sclerosis. Neurology 2009;72:1077-1082.

23. De Seze J, Blanc F, Jeanjean L, et al. Optical coherence tomography in neuromyelitis optica. Arch Neurol 2008;65:920-923.

24. Wang J-K, Kardon RH, Kupersmith MJ, Garvin MK. Automated quantification of volumetric optic disc swelling in papilledema using spectral-domain optical coherence tomography. Invest Ophthalmol Vis Sci 2012;53:4069-4075.

25. Skau M, Yri H, Sander B, Gerds TA, Milea D, Jensen R. Diagnostic value of optical coherence tomography for intracranial pressure in idiopathic intracranial hypertension. Graefes Arch Clin Exp Ophthalmol 2013;251:567-574.

26. Rebolleda G, Muñoz-Negrete FJ. Follow-up of mild papilledema in idiopathic intracranial hypertension with optical coherence tomography. Invest Ophthalmol Vis Sci 2009;50:5197-5200.

27. Sarac O, Tasci YY, Gurdal C, Can I. Differentiation of optic disc edema from optic nerve head drusen with spectral-domain optical coherence tomography. J Neuroophthalmol 2012;32:207-211.

28. Purvin V, Sundaram S, Kawasaki A. Neuroretinitis: review of the literature and new observations. J Neuroophthalmol 2011;31:58-68.

29. Ling JD, Chao D, Al Zubidi N, Lee AG. Big red flags in neuro-ophthalmology. Can J Ophthalmol 2013;48:3-7. 
30. Wade NK, Levi L, Jones MR, Bhisitkul R, Fine L, Cunningham ET. Optic disk edema associated with peripapillary serous retinal detachment: an early sign of systemic Bartonella henselae infection. Am J Ophthalmol 2000;130:327-334.

31. Habot-Wilner Z, Zur D, Goldstein M, et al. Macular findings on optical coherence tomography in cat-scratch disease neuroretinitis. Eye 2011;25:1064-1068.

32. Kitamei H, Suzuki Y. Retinal angiography and optical coherence tomography disclose focal optic disc vascular leakage and lipid-rich fluid accumulation within the retina in a patient with. J Neuroophthalmol 2009;29:203-207.

33. Chang L, El-Dairi MA, Frempong TA, et al. Optical coherence tomography in the evaluation of neurofibromatosis type-1 subjects with optic pathway gliomas. J AAPOS 2010;14:511-517.

34. Avery RA, Liu GT, Fisher MJ, et al. Retinal nerve fiber layer thickness in children with optic pathway gliomas. Am J Ophthalmol 2011;151:542-549.

\section{ACKNOWLEDGMENT}

The authors thank Marian Jabbour for contribution in the design of figure 1 .

\section{AUTHOR CONTRIBUTIONS}

Dr. Maldonado: design, conceptualization, and writing. Dr. Mettu: design, conceptualization, and writing. Dr. El-Dairi: drafting and revising manuscript for intellectual content. Dr. Bhatti: conceptualization, drafting and revising manuscript for intellectual content.

\section{STUDY FUNDING}

Supported by a Duke Eye Center Department grant from Research to Prevent Blindness, Inc.

\section{DISCLOSURES}

R.S. Maldonado and P. Mettu report no disclosures. M. El-Dairi serves as a consultant for Prana Biotechnology and receives research support from The Knights Templar. M. Bhatti has received funding for travel from and serves on a scientific advisory board, as a consultant, and on the speakers' bureaus for Novartis and serves on the editorial board of Journal of Neuro-Ophthalmology. Full disclosure form information provided by the authors is available with the full text of this article at Neurology.org/cp.

\section{Related articles from AAN physician and patient resources}

\section{Neurology ${ }^{\circledR}$ Clinical Practice}

Fingolimod and macular edema: Pathophysiology, diagnosis, and management October 2014;4:402-409.

\section{Neurology ${ }^{\circledR} \quad$ - Neurology.org}

Retinal pathology in Susac syndrome detected by spectral-domain optical coherence tomography August 18, 2015;85:610-618.

Retinal pathology in idiopathic moyamoya angiopathy detected by optical coherence tomography August 11, 2015;85:521-527.

\section{Continuum ${ }^{\circledR}$ - ContinuumJournal.com}

Diagnostic Approach to Vision Loss

August 2014;20:785-815.

The Importance of Visual Field Testing in Idiopathic Intracranial Hypertension August 2014;20:1067-1074. 